\title{
FARELO DE ARROZ: CAPACIDADE ANTIOXIDANTE DE FRAÇÕES RICAS EM ORIZANÓIS
}

\author{
JOSIANE FREITAS CHIM* \\ RUI CARLOS ZAMBIAZI** \\ ANGELITAMACHADO LEITÃO***
}

\begin{abstract}
Avaliou-se a capacidade antioxidante de frações ricas em orizanol extraídas de farelo de arroz, mediante solventes orgânicos, testadas em óleo vegetal rico em ácidos graxos poliinsaturados. Amostras de farelo de arroz foram imersas $24 \mathrm{~h}$ em diferentes solventes orgânicos (clorofórmio; clorofórmio: hexano, 1:1v/v; hexano; e acetona) e o extrato orgânico filtrado e concentrado em evaporador a vácuo. Alíquotas dos extratos foram aplicadas em óleos de girassol armazenados sob aquecimento a $60^{\circ} \mathrm{C}$ na ausência de luz e a $35^{\circ} \mathrm{C}$ na presença de luz, avaliando-se sua ação antioxidante mediante análise de índice de peróxido. A partir do terceiro dia de aquecimento das amostras, na presença e ausência de luz, os extratos exerceram ação retardadora no processo oxidativo do óleo de girassol. Em ambos os testes, o extrato obtido com clorofórmio exerceu maior ação antioxidante, seguido pelo obtido com clorofórmio:hexano e dos extraídos com hexano e com acetona. Conclui-se que todos os extratos obtidos do farelo de arroz pelo uso de solventes orgânicos apresentaram ação antioxidante, tanto na ausência quanto na presença de luz, e que o extraído com clorofórmio apresentou maior capacidade antioxidante.
\end{abstract}

PALAVRAS-CHAVE: ORIZANOL-EXTRAÇÃO; SOLVENTES ORGÂNICOS; OXIDAÇÃO; ANTIOXIDANTE.

* Química de Alimentos, doutoranda do Curso de Pós-graduação em Ciência e Tecnologia Agroindustrial, Universidade Federal de Pelotas (UFPel), Capão do Leão (RS) Brasil (e-mail: josianechim@gmail.com).

** Professor Titular, PhD em Food of Science, Departamento de Ciência dos Alimentos, UFPel, Capão do Leão (RS) Brasil (e-mail: zambiazi@ufpel. tche.br).

*** Química de Alimentos, mestranda do curso de Pós-graduação em Ciência e Tecnologia Agroindustrial, UFPel, Capão do Leão RS Brasil. 


\section{INTRODUÇÃO}

A região de Pelotas no estado do Rio Grande do Sul, área característica de produção de arroz, conta com várias indústrias de beneficiamento desse grão. Um dos subprodutos do seu beneficiamento é o farelo de arroz, parcialmente aproveitado pela indústria de extração de óleo comestível.

Vários estudos destacam a importância do farelo de arroz como fonte de antioxidantes naturais (WANG e LUH, 1991). A fração lipídica do farelo de arroz contém orizanóis, tocoferóis, esteróis e lecitina. Os orizanóis, presentes no farelo de arroz em cerca de $3.000 \mathrm{mg} / \mathrm{Kg}$, têm sido apontados como compostos com poder antioxidante, que alcançam elevado valor comercial em países como o Japão. Sua utilização tem sido ainda bastante restrita devido principalmente ao alto custo da fase de purificação das frações lipídicas em componentes específicos. A eficiência dos orizanóis em termos de atividade antioxidante ainda é pouco pesquisada, assim como sua ação em diferentes tipos de alimentos ricos em gordura (GONG-YUANSSHENG e YAO-HUIYUAN, 2001; ZHIMIN et al., 2001; PATEL e NAIKSN, 2004).

O $\gamma$-orizanol, mistura de ésteres do ácido ferúlico com esterol ou alcóois triterpênicos, apresenta ponto de fusão na faixa de 135 - 137ํㅡ (THE MERCK INDEX, 1996). Cicloartenil ferulato, 24-metilenocicloartonil ferulato, campesteril ferulato e $\beta$-sistosteril ferulato são componentes majoritários da mistura que inclui também outros componentes como, estigmastaril ferulato (EVERSHED et al., 1988; GODBER et al., 2001; CHEN et al., 2005).

A atividade antioxidante do $\gamma$-orizanol pode ser atribuída principalmente ao ácido ferúlico, que se apresenta esterificado com esteróis de plantas. A esterificação aumenta o potencial antioxidante, provendo acesso molecular aos componentes hidrofóbicos. Esses são mais susceptíveis à destruição celular oxidativa, pois o potencial antioxidante aumenta com a extensão da hidroxilação dos anéis aromáticos (TSUNO..., 1995; ZHIMIN, et al., 2001).

A estrutura química da maioria dos componentes do $\gamma$-orizanol, encontrados no óleo de farelo de arroz, é similar a do colesterol. Tal 
similaridade pode ser responsável pelo efeito de diminuição da absorção e síntese do colesterol (ROGERS et al., 1993), dos níveis do colesterol hepático e aumento da secreção fecal de ácidos biliares (ROGERS et al., 1993; ORTHOEFER, 1996; PATEL e NAIKSN, 2004).

A comprovação da ação antioxidante das frações de $\gamma$-orizanol permitiria seu uso em alimentos, visando reduzir a adição de antioxidantes artificiais como butilhidroxianisol (BHA) e butilhidroxitolueno (BHT). Tais substâncias, devido aos seus efeitos cancerígenos, representam fatores de preocupação para a indústria de alimentos (IQBAL et al., 2005).

Considerando a perspectiva de incremento de uso de antioxidante natural, a obtenção das frações do $\gamma$-orizanol poderia representar subprodutos com alto valor econômico para a indústria processadora de óleos vegetais (ZHIMIN et al., 2001; IQBAL et al., 2005). Assim há interesse industrial na obtenção de frações contendo orizanóis, mesmo com baixo grau de pureza, mas que apresentem ação antioxidante comprovada.

Este estudo teve como objetivo extrair frações ricas em orizanóis, mediante processos economicamente acessíveis à realidade das indústrias da região de Pelotas, e testá-las quanto as suas propriedades antioxidantes em produtos com alta instabilidade.

\section{MATERIAL E MÉTODOS}

\subsection{MATERIAL}

Foram utilizados farelo de arroz, obtido de indústria local (Cidade de Pelotas), e os solventes orgânicos clorofórmio, acetona e hexano de marcas comerciais. Para a realização dos testes de ação antioxidante utilizou-se óleo de girassol, adquirido no comércio local.

\subsection{PROCESSO DE EXTRAÇÃO E AVALIAÇÃO DA CAPACIDADE ANTIOXIDANTE}

A extração e a avaliação da capacidade antioxidativa das frações seguiu o fluxograma apresentado na Figura 1. 


\title{
FIGURA 1 - PROCESSO DE EXTRAÇÃO DE FRAÇÕES E AVALIAÇÃO DA CAPACIDADE ANTIOXIDANTE
}

\author{
FARE LO DE ARROZ \\ $\downarrow$ \\ IMER SÃO EM SOLVENTES ORGANICOS \\ 1 \\ CONCENTRAÇÄO D AS FRAÇÖES \\ $\downarrow$ \\ APLIC AÇAO DAS FRAÇOES EM OLEOS \\ VE GETAIS \\ $\downarrow$ \\ AQUE CIMENTO DOS OLEOS VEGET ANS \\ - EM PRE SENÇA DE LUZ a $60^{\circ} \mathrm{C}$ \\ - NAAUSEENCIA DE LUZ a $35^{\circ} \mathrm{C}$ \\ 1
}

\section{ANÁLISES QUIIMICAS}

Para a extração das frações ricas em orizanóis utilizou-se metodologia adaptada de WAN (1995), sendo o farelo de arroz (500 g) colocado em imersão em diferentes solventes orgânicos $(1 \mathrm{~L})$ : clorofórmio, acetona, hexano e hexano: clorofórmio $(1: 1, v / v)$ durante 24 horas. Ao final da imersão, as soluções foram filtradas e os sobrenadantes concentrados a vácuo $\left(35^{\circ} \mathrm{C}\right)$. Alíquotas de $2 \mathrm{~mL}$ de cada uma das quatro frações obtidas foram adicionadas em $75 \mathrm{~mL}$ de óleo de girassol. 
Os óleos foram aquecidos a $60^{\circ} \mathrm{C}$ na ausência de luz e a $35^{\circ} \mathrm{C}$ na presença de luz, durante 14 dias, segundo a metodologia "Schall Oven Test" (WAN, 1995). O aquecimento das amostras foi realizado em estufa convencional de laboratório (com ajuste de temperatura) e para o fornecimento de luz acoplou-se lâmpada de 60/W na posição central da parte superior interna da estufa.

Amostras foram removidas para a determinação do índice de peróxido, a cada dois dias, parâmetro utilizado para avaliar o grau de oxidação. Preparou-se prova em branco com $75 \mathrm{~mL}$ de óleo de girassol, submetida às mesmas condições.

O experimento foi realizado em duplicata e os valores do índice de peróxido referem-se a média de três determinações.

\subsection{ANÁLISES QUÍMICAS}

Foram realizadas análises de índice de peróxido, segundo a metodologia da AOCS (1991), nas diferentes amostras e durante o período de aquecimento. Os tempos pré-estabelecidos foram: tempo zero (refere-se às amostras antes de serem submetidas ao aquecimento), $3^{\circ}, 5^{\circ}, 7^{\circ}, 9^{\circ}, 11^{\circ}$ e $14^{\circ}$ dia de aquecimento.

\subsection{ANÁLISE ESTATÍSTICA}

Os resultados foram avaliados pela análise de variância ANOVA e quando apresentaram diferença significativa de médias submetidos ao teste de Tukey (nível de $5 \%$ de probabilidade), usando-se o programa Statistica 6.0 (STATSOFT, 1998).

\section{RESULTADOS E DISCUSSÃO}

As frações resultantes do processo de extração pelos diferentes solventes e da concentração a vácuo apresentaram volumes diferenciados (Tabela1).

A fração obtida com a mistura clorofórmio: hexano (1:1, v/v) evidenciou volume final cerca de $20 \%$ superior às demais frações, chegando a $40 \%$ em relação ao uso de clorofórmio. 
A Tabela 2 demonstra os dados de índice de peróxido para as amostras submetidas ao tratamento na ausência de luz.

Com base nos dados experimentais todas as amostras apresentaram índice de peróxido abaixo de 4 no ponto zero, não havendo influência aparente da ação dos diferentes extratos sobre o grau de oxidação das amostras.

\section{TABELA 1 - VOLUME DAS FRAÇÕES ANTIOXIDANTES APÓS A} CONCENTRAÇÃO

\begin{tabular}{lc}
\hline \multicolumn{1}{c}{ SOLVENTE DE } & VOLUME D A \\
EXTRAÇÃO & FRAÇÃO (m L) \\
\hline CIorofórmio: Hexano $(1: 1, v / v)$ & 45,0 \\
Acetona & 36,2 \\
Hexano & 36,2 \\
Clorofórmio & 25,5 \\
\hline
\end{tabular}

Observou-se o início do processo de oxidação em todas as amostras ao terceiro dia de aquecimento, indicando ação mais eficaz na retenção do processo oxidativo das frações extraídas com clorofórmio, seguidas pelas frações extraídas pela mistura hexano: clorofórmio $(1: 1, \mathrm{v} / \mathrm{v})$. Essa tendência foi observada até o quinto dia de aquecimento.

\section{TABELA 2-ÍNDICE DE PERÓXIDO (meqg/1000 g) DAS AMOSTRAS SUBMETIDAS A $60^{\circ} \mathrm{C}$ NA AUSÊNCIA DE LUZ}

\begin{tabular}{|c|c|c|c|c|c|}
\hline \multirow{2}{*}{ Dias } & \multicolumn{5}{|c|}{ Índice de peróxido } \\
\hline & Padrã o* & Acetona & $\mathrm{Hexan0^{* }}$ & $\begin{array}{c}\text { Hexano: } \\
\text { Clor ofór mio } \\
\text { * }\end{array}$ & Clorofórmio* \\
\hline $0^{\star \star}$ & $3.98 \mathrm{a}$ & $3.95 a$ & $3.92 a$ & $3.95 a$ & $3.98 \mathrm{a}$ \\
\hline 3 & $16.13 \mathrm{c}$ & $15.25 \mathrm{a}$ & $14.99 \mathrm{~b}$ & $12.87 \mathrm{~d}$ & $9.87 \mathrm{e}$ \\
\hline 5 & $37.33 \mathrm{~b}$ & $35.29 b$ & $36.60 a$ & $34.94 \mathrm{c}$ & $27.70 \mathrm{~d}$ \\
\hline 7 & $55.27 d$ & $54.44 b$ & $54.36 \mathrm{c}$ & $54.03 \mathrm{a}$ & $48.46 \mathrm{e}$ \\
\hline 9 & $103.73 a$ & $102.04 \mathrm{~b}$ & $100.23 d$ & $100.86 \mathrm{c}$ & $90.45 \mathrm{e}$ \\
\hline 11 & $136.71 \mathrm{a}$ & $130.77 b$ & $128.94 \mathrm{c}$ & $125.08 \mathrm{~d}$ & $120.71 \mathrm{e}$ \\
\hline 14 & $164.93 \mathrm{a}$ & $156.16 \mathrm{c}$ & $159.53 \mathrm{~b}$ & $153.77 \mathrm{~d}$ & $146.97 \mathrm{e}$ \\
\hline
\end{tabular}

*Solventes utilizados para o processo de extração das frações ricas em orizanóis submetidas ao aquecimento. **ponto zero = análises dos óleos de girassol antes de serem submetidos ao aquecimento. Letras diferentes na mesma linha evidenciam diferença significativa ao nível de $5 \%$ de probabilidade pelo teste de Tukey. 
A fração resultante do processo de extração com clorofórmio continuou apresentando eficiência significativamente superior às demais, quanto ao retardamento das reações oxidativas, em todo o período de aquecimento.

Durante os 14 dias de monitoramento, a amostra padrão (sem extratos) apresentou os maiores valores de índice de peróxido. Tal fato indica maior grau de oxidação em relação às amostras contendo os extratos, principalmente após o décimo primeiro dia de aquecimento.

A Tabela 3 demonstra os dados referentes ao índice de peróxido nas amostras submetidas ao aquecimento a $35^{\circ} \mathrm{C}$ na presença de luz.

\section{TABELA 3 - ÍNDICE DE PERÓXIDO (meqg/1000 g) DOS ÓLEOS VEGETAIS SUBMETIDOS AO AQUECIMENTO NA PRESENÇA DE LUZ A 35C}

\begin{tabular}{cccccc}
\hline & \multicolumn{5}{c}{ Índice de peróxido } \\
\cline { 2 - 6 } Dias & Padrão* & Acetona* $^{*}$ & Hexano* & Hexano:Clorofórmi o* & Clorofórmio* $^{*}$ \\
\hline $0^{\star *}$ & $3.98 \mathrm{a}$ & $3.95 \mathrm{a}$ & $3.92 \mathrm{a}$ & $3.95 \mathrm{a}$ & $3.98 \mathrm{a}$ \\
\hline 3 & $27.58 \mathrm{c}$ & $28.23 \mathrm{~b}$ & $28.84 \mathrm{a}$ & $26.03 \mathrm{~d}$ & $17.64 \mathrm{e}$ \\
\hline 5 & $40.85 \mathrm{a}$ & $39.97 \mathrm{~b}$ & $40.83 \mathrm{a}$ & $38.13 \mathrm{c}$ & $24.45 \mathrm{~d}$ \\
\hline 7 & $53.69 \mathrm{c}$ & $59.70 \mathrm{a}$ & $57.89 \mathrm{~b}$ & $52.64 \mathrm{~d}$ & $36.21 \mathrm{e}$ \\
\hline 9 & $66.45 \mathrm{~b}$ & $65.30 \mathrm{c}$ & $67.45 \mathrm{a}$ & $64.93 \mathrm{~d}$ & $45.07 \mathrm{e}$ \\
\hline 11 & $111.25 \mathrm{~b}$ & $107.71 \mathrm{c}$ & $106.21 \mathrm{~d}$ & $111.71 \mathrm{a}$ & $84.45 \mathrm{e}$ \\
\hline 14 & $314.49 \mathrm{~b}$ & $274.39 \mathrm{c}$ & $251.65 \mathrm{~d}$ & $392.37 \mathrm{a}$ & $243.51 \mathrm{e}$ \\
\hline
\end{tabular}

*Solventes utilizados para o processo de extração das frações ricas em orizanóis submetidas ao aquecimento. ${ }^{* *}$ ponto zero = análises dos óleos de girassol antes de serem submetidos ao aquecimento. Letras diferentes na mesma linha evidenciam diferença significativa ao nível de $5 \%$ de probabilidade pelo teste de Tukey.

A fração extraída com clorofórmio nas condições de aquecimento na presença de luz a $35^{\circ} \mathrm{C}$ apresentou em todo o período de monitoramento os menores valores de índice de peróxido, revelando maior capacidade antioxidativa. As demais frações seguiram praticamente o mesmo comportamento da amostra padrão até o $11^{\circ}$ dia quanto ao grau de oxidação. Após esse dia, a fração extraída com hexano: clorofórmio $(1: 1, v / v)$ revelou inclusive índice de oxidação superior ao padrão. Já as demais frações demonstraram pequena atividade antioxidante. 
Pelos dados das Tabelas 2 e 3, todas as amostras apresentaram processos oxidativos mais acelerados durante os primeiros dias de aquecimento quando estocadas na presença de luz (35으). Essa tendência se inverteu, entre o quinto e o sétimo dia, verificando-se maior intensidade dos processos oxidadtivos nas amostras estocadas na ausênca de luz $\left(60^{\circ} \mathrm{C}\right)$.

Constatou-se que a incidência de luz, mesmo em temperaturas mais baixas, exerce efeito mais marcante na aceleração de processos oxidativos no estádio inicial do que o aquecimento em ambiente isento de luz. Estes dados comprovam a presença de compostos fotossensores no óleo de girassol, provavelmente residuais de clorofila, que atuam como catalisadores em reações de oxidação de ácidos graxos insaturados (ZAMBIAZI, 1997).

\section{CONCLUSÃO}

Os solventes orgânicos utilizados no estudo demonstraram-se eficazes no processo de extração de frações ricas em compostos antioxidantes. As frações obtidas com clorofórmio apresentaram maior capacidade antioxidativa nas duas condições estudadas. O aquecimento na presença de luz provocou aceleração do processo oxidativo no período inicial de estocagem, enquanto que o aquecimento na ausência de luz induziu maior oxidação das amostras em períodos mais longos de aquecimento.

\section{Abstract \\ RICE BRAN: ANTIOXIDANT CAPACITY OF ORYZANOLS RICH FRACTIONS}

The antioxidant capacity of oryzanol rich fractions extracted from rice bran was evaluated by means of organic solvents, tested in vegetable oil rich in polyunsaturated fatty acids. Samples of rice bran were immerged $24 \mathrm{~h}$ in different organic solvents (chloroform; chloroform: hexane, 1:1v/v; hexane; and acetone) and the organic extract was filtered and concentrated in a vacuum evaporator. Aliquots of the extracts were applied in sunflower oils stored at $60^{\circ} \mathrm{C}$ in light absence and at $35^{\circ} \mathrm{C}$ in the light presence, being evaluated its antioxidant action by means of analysis of peroxide index. Starting from the third day of heating of the samples, in the presence and light absence, the extracts promoted a retarded action in the oxidative process of the sunflower oil. In both tests, the extract obtained with chloroform supported larger antioxidant action, proceeded for the obtained with chloroform:hexane and of the 
extracted with hexane and with acetone. It is concluded that all the obtained extracts of the rice bran by the use of organic solvents presented antioxidant action, as much in the absence as in the light presence, and that the extracted with chloroform presented higher antioxidant capacity.

KEY-WORDS: ORYZANOL-EXTRACTION; ORGANIC SOLVENTS; OXIDATION; ANTIOXIDANT.

\section{REFERÊNCIAS}

1 AOCS. American Oil Chemists' Society. Official and tentative methods of the AOCS. Champaign, IL, 1991.

2 CHEN, M.N.; BERGMAN, C.J. A rapid procedure for analyzing rice bran tocopherol, tocotrienol and $\gamma$-oryzanol contents. Journal of Food Composition and Analysis, v. 18, p. 139-151, 2005.

3 EVERSHED, R.P.; SPOONER, N.; PRESCOTT,M.C.; GOALD, L.J. Isolation and characterization of intact steryl ferulate from seeds. Journal of Chromatography, Amsterdam, v. 440, p. 2335, 1988.

4 GODBER, J.S.; ZHIMIN, X. Antioxidant activities of major components of gamma-oryzanol from rice bran using a linolenic acid model. Journal of the American Oil Chemists' Society, v. 76, p. 645-649, 2001.

5 GONG-YUANSSHENG, Y.; YAO-HUIYUAN, H. Purification and identification of gamma-oryzanol from rice bran. Journal of the Chinese Cereals and Oils Association, v. 16, p.30-34, 2001.

6 IQBAL, S.; BHANGER, M.I.; ANWAR, F. Antioxidant properties and components of some commercially available varieties of rice bran in Pakistan. Food Chemistry On- line. Disponível: www.elselvier.com/locate/foodchemistry. 2005. Acesso: 23 nov. 2005.

7 IAL. Instituto Adolfo Lutz. Normas analíticas do Instituto Adolfo Lutz. São Paulo, 1985. v.1. 
8 ORTHOEFER, F. T. Rice bran oil: healthy lipid source. Food Technology, Chicago, v. 50, n. 12, p. $62-64,1996$.

9 PATEL, M.; NAIKSN, N. $\gamma$-oryzanol from rice bran oil a review. J. Scientific \& Ind. Research, v. 63, v.7, p. 569 - 578, Jul. 2004.

10 ROGERS, E. J.; RICE, S.M.; NICOLOSI, R.J.; CARPENTER, D.R.; Mc CLELLAND, C. A.; ROMANCZYK, L. J. Identification and quantitation of $\gamma$-oryzanol components and simultaneous assessment of tocols and rice bran oil. Journal of the American Oil Chemists Society, Champaign, v. 70, n. 3, p. 301-307, 1993.

11 STATSOFT Inc. Statistica. Tulsa, 1998. Version 6.0.

12 THE MERCK INDEX. $12^{\text {th }}$ ed. New York: Merck Research Laboratories, 1996. p. 1181.

13 TSUNO RICE FINE CHEMICAL CO., LTD. $\boldsymbol{\gamma}$-oryzanol, a naturally derived food antioxidant. Wakayama, Japan, 1995. 8 p.

14 WAN, P. J. Accelerated stability methods. In: WARNER, K.; ESKIN, N. A. (eds). Methods to assess quality and stability of oils and fat-containing foods, Champaign, FL: AOCS Press, 1995. p. 179-189.

15 WANG, C.Y.; LUH, B. S. Harvest, drying, and storage of rough rice. In: LUH, B. S. Rice production. $2^{\text {nd }}$ ed. New York: Van Nostrand Reinhold, 1991. p. 311-345.

16 ZAMBIAZI, R.C.Z. The role of endogenous lipid components on vegetable oil stability. Winnipeg, 1997. $153 \mathrm{p}$. Tese (Doutorado em Food and Nutritional Science) Foods and Nutritional Sciences Interdepartamental Program, University of Manitoba Canadá.

17 ZHIMIN, X.; HUA, N.; GODBER, J. S. Antioxidant activity of tocopherols, tocotrienols, and $\gamma$-oryzanol components from rice bran against cholesterol oxidation accelerated by 2,2'-azobis (2methylpropionamidine) dihydrochloide. J. Agric. Food Chem., v. 49, p. $2077-2081,2001$. 